\title{
Unsupervised Discovery of Gendered Language through Latent-Variable Modeling
}

\author{
Alexander Hoyle $^{\partial}$ Lawrence Wolf-Sonkin $^{\int}$ Hanna Wallach $^{3}$ \\ Isabelle Augenstein $^{\text {? }}$ Ryan Cotterell ${ }^{\mathrm{h}}$ \\ 'University College London, London, UK

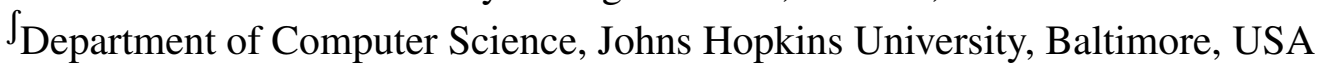 \\ ${ }^{3}$ Microsoft Research, New York City, USA

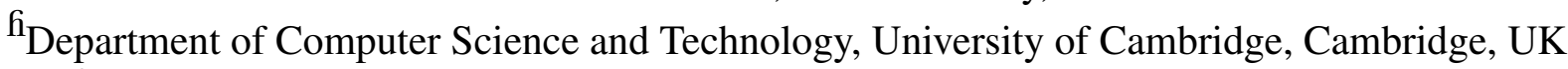 \\ ${ }^{\mathrm{P}}$ Department of Computer Science, University of Copenhagen, Copenhagen, Denmark \\ alexander.hoyle.17@ucl.ac.uk, lawrencews@jhu.edu \\ hanna@dirichlet.net, augenstein@di.ku.dk, rdc42@cam.ac.uk
}

\begin{abstract}
Studying the ways in which language is gendered has long been an area of interest in sociolinguistics. Studies have explored, for example, the speech of male and female characters in film and the language used to describe male and female politicians. In this paper, we aim not to merely study this phenomenon qualitatively, but instead to quantify the degree to which the language used to describe men and women is different and, moreover, different in a positive or negative way. To that end, we introduce a generative latent-variable model that jointly represents adjective (or verb) choice, with its sentiment, given the natural gender of a head (or dependent) noun. We find that there are significant differences between descriptions of male and female nouns and that these differences align with common gender stereotypes: Positive adjectives used to describe women are more often related to their bodies than adjectives used to describe men.
\end{abstract}

\section{Introduction}

Word choice is strongly influenced by genderboth that of the speaker and that of the referent (Lakoff, 1973). Even within 24 hours of birth, parents describe their daughters as beautiful, pretty, and cute far more often than their sons (Rubin et al., 1974). To date, much of the research in sociolinguistics on gendered language has focused on laboratory studies and smaller corpora (McKee and Sherriffs, 1957; Williams and Bennett, 1975; Baker, 2005); however, more recent work has begun to focus on larger-scale datasets (Pearce, 2008; CaldasCoulthard and Moon, 2010; Baker, 2014; Norberg, 2016). These studies compare the adjectives (or

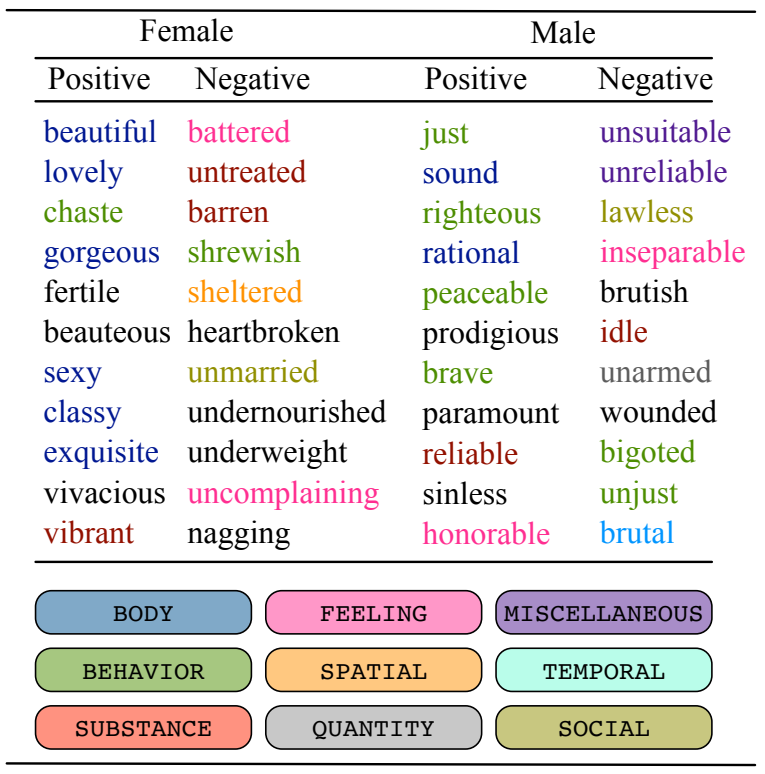

Figure 1: Adjectives, with sentiment, used to describe men and women, as represented by our model. Colors indicate the most common sense of each adjective from Tsvetkov et al. (2014); black indicates out of lexicon. Two patterns are immediately apparent: positive adjectives describing women are often related to their bodies, while positive adjectives describing men are often related to their behavior. These patterns hold generally and the differences are significant (see $\S 4)$.

verbs) that modify each noun in a particular gendered pair of nouns, such as boy-girl, aggregated across a given corpus. We extend this line of work by instead focusing on multiple noun pairs simultaneously, modeling how the choice of adjective (or verb) depends on the natural gender ${ }^{1}$ of the head

\footnotetext{
${ }^{1}$ A noun's natural gender is the implied gender of its referent (e.g., actress refers to woman). We distinguish natural
} 
(or dependent) noun, abstracting away the noun form. To that end, we introduce a generative latentvariable model for representing gendered language, along with sentiment, from a parsed corpus. This model allows us to quantify differences between the language used to describe men and women.

The motivation behind our approach is straightforward: Consider the sets of adjectives (or verbs) that attach to gendered, animate nouns, such as man or woman. Do these sets differ in ways that depend on gender? For example, we might expect that the adjective Baltimorean attaches to man roughly the same number of times as it attaches to woman, controlling for the frequency of man and woman. ${ }^{2}$ But this is not the case for all adjectives. The adjective pregnant, for example, almost always describes women, modulo the rare times that men are described as being pregnant with, say, emotion. Arguably, the gendered use of pregnant is benign - it is not due to cultural bias that women are more often described as pregnant, but rather because women bear children. However, differences in the use of other adjectives (or verbs) may be more pernicious. For example, female professors are less often described as brilliant than male professors (Storage et al., 2016), likely reflecting implicit or explicit stereotypes about men and women.

In this paper, we therefore aim to quantify the degree to which the language used to describe men and women is different and, moreover, different in a positive or negative way. Concretely, we focus on three sociolinguistic research questions about the influence of gender on adjective and verb choice:

Q1 What are the qualitative differences between the language used to describe men and women? For example, what, if any, are the patterns revealed by our model? Does the output from our model correlate with previous human judgments of gender stereotypes?

Q2 What are the quantitative differences between the language used to describe men and women? For example, are adjectives used to describe women more often related to their bodies than adjectives used to describe men? Can we quantify such patterns using existing semantic resources (Tsvetkov et al., 2014)?

gender from grammatical gender because the latter does not necessarily convey anything meaningful about the referent.

${ }^{2}$ Men are written about more often than women. Indeed, the corpus we use exhibits this trend, as shown in Tab. 1.

\begin{tabular}{lrlr}
\hline \multicolumn{2}{c}{ Female } & \multicolumn{2}{c}{ Male } \\
\hline other & 2.2 & other & 6.8 \\
daughter & 1.4 & husband & 1.8 \\
lady & 2.4 & king & 2.1 \\
wife & 3.3 & son & 2.9 \\
mother & 4.2 & father & 4.2 \\
girl & 5.1 & boy & 5.1 \\
woman & 11.5 & man & 39.9 \\
\hline Total & 30.2 & & 62.7 \\
\hline
\end{tabular}

Table 1: Counts, in millions, of male and female nouns present in the corpus of Goldberg and Orwant (2013).

\section{Q3 Does the overall sentiment of the language} used to describe men and women differ?

To answer these questions, we introduce a generative latent-variable model that jointly represents adjective (or verb) choice, with its sentiment, given the natural gender of a head (or dependent) noun. We use a form of posterior regularization to guide inference of the latent variables (Ganchev et al., 2010). We then use this model to study the syntactic $n$-gram corpus of (Goldberg and Orwant, 2013).

To answer Q1, we conduct an analysis that reveals differences between descriptions of male and female nouns that align with common gender stereotypes captured by previous human judgements. When using our model to answer Q2, we find that adjectives used to describe women are more often related to their bodies (significant under a permutation test with $p<0.03$ ) than adjectives used to describe men (see Fig. 1 for examples). This finding accords with previous research (Norberg, 2016). Finally, in answer to Q3, we find no significant difference in the overall sentiment of the language used to describe men and women.

\section{What Makes this Study Different?}

As explained in the previous section, many sociolinguistics researchers have undertaken corpusbased studies of gendered language. In this section, we therefore differentiate our approach from these studies and from recent NLP research on gender biases in word embeddings and co-reference systems.

Syntactic collocations and noun types. Following the methodology employed in previous sociolinguistic studies of gendered language, we use syntactic collocations to make definitive claims about gendered relationships between words. This approach stands in contrast to bag-of-words analyses, where information about gendered relationships must be indirectly inferred. By studying the 
adjectives and verbs that attach to gendered, animate nouns, we are able to more precisely quantify the degree to which the language used to describe men and women is different. To date, much of the corpus-based sociolinguistics research on gendered language has focused on differences between the adjectives (or verbs) that modify each noun in a particular gendered pair of nouns, such as boygirl or man-woman (e.g., Pearce (2008); CaldasCoulthard and Moon (2010); Norberg (2016)). To assess the differences, researchers typically report top collocates ${ }^{3}$ for one word in the pair, exclusive of collocates for the other. This approach has the effect of restricting both the amount of available data and the claims that can be made regarding gendered nouns more broadly. In contrast, we focus on multiple noun pairs (including plural forms) simultaneously, modeling how the choice of adjective (or verb) depends on the natural gender of the head (or dependent) noun, abstracting away the noun form. As a result, we are able to make broader claims.

The corpus of Goldberg and Orwant (2013). To extract the adjectives and verbs that attach to gendered, animate nouns, we use the corpus of Goldberg and Orwant (2013), who ran a then-state-of-the-art dependency parser on 3.5 million digitalized books. We believe that the size of this corpus (11 billion words) makes our study the largest collocational study of its kind. Previous studies have used corpora of under one billion words, such as the British National Corpus (100 million words) (Pearce, 2008), the New Model Corpus (100 million words) (Norberg, 2016), and the Bank of English Corpus (450 million words) (Moon, Rosamund, 2014). By default, the corpus of Goldberg and Orwant (2013) is broken down by year, but we aggregate the data across years to obtain roughly 37 million noun-adjectives pairs, 41 million NSUBJ-verb pairs, and 14 million DOBJ-verb pairs. We additionally lemmatize each word. For example, the noun stewardesses is lemmatized to a set of lexical features consisting of the genderless lemma STEWARD and the morphological features +FEM and + PL. This parsing and lemmatization process is illustrated in Fig. 2.

Quantitative evaluation. Our study is also quantitative in nature: we test concrete hypotheses about differences between the language used to describe men and women. For example, we test whether

\footnotetext{
${ }^{3}$ Typically ranked by the log of the Dice coefficient.
}

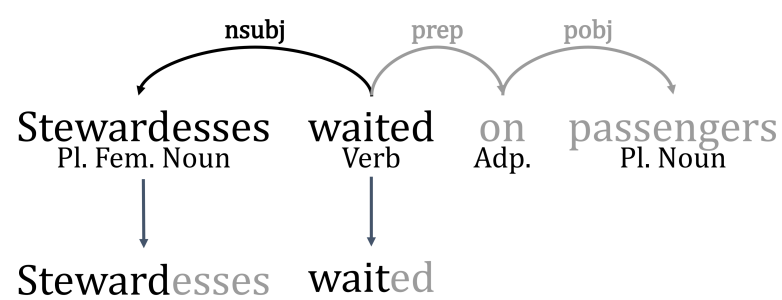

Figure 2: An example sentence with its labeled dependency parse (top) and lemmatized words (bottom).

women are more often described using adjectives related to their bodies and emotions. This quantitative focus differentiates our approach from previous corpus-based sociolinguistics research on gendered language. Indeed, in the introduction to a special issue on corpus methods in the journal Gender and Language, Baker (2013) writes, "while the term corpus and its plural corpora are reasonably popular within Gender and Language (occurring in almost $40 \%$ of articles from issues 1-6), authors have mainly used the term as a synonym for 'data set' and have tended to carry out their analysis by hand and eye methods alone." Moreover, in a related paper on extracting gendered language from word embeddings, Garg et al. (2018) lament that "due to the relative lack of systematic quantification of stereotypes in the literature [... they] cannot directly validate [their] results." For an overview of quantitative evaluation, we recommend Baker (2014).

Speaker versus referent. Many data-driven studies of gender and language focus on what speakers of different genders say rather than differences between descriptions of men and women. This is an easier task - the only annotation required is the gender of the speaker. For example, Ott (2016) used a topic model to study how word choice in tweets is influenced by the gender of the tweeter; Schofield and Mehr (2016) modeled gender in film dialog; and, in the realm of social media analysis, Bamman et al. (2014) discussed stylistic choices that enable classifiers to distinguish between tweets written by men versus women.

Model versus data. Recent NLP research has focused on gender biases in word embeddings (Bolukbasi et al., 2016; Zhao et al., 2017) and co-reference systems (Zhao et al., 2018; Rudinger et al., 2018). These papers are primarily concerned with mitigating biases present in the output of machine learning models deployed in the real world (O'Neil, 2016). For example, Bolukbasi et al. (2016) used pairs 
of gendered words, such as she-he, to mitigate unwanted gender biases in word embeddings. Although it is possible to rank the adjectives (or verbs) most aligned with the embedding subspace defined by a pair of gendered words, there are no guarantees that the resulting adjectives (or verbs) were specifically used to describe men or women in the dataset from which the embeddings were learned. In contrast, we use syntactic collocations to explicitly represent gendered relationships between individual words. As a result, we are able make definitive claims about these relationships, thereby enabling us to answer sociolinguistic research questions. Indeed, it is this sociolinguistic focus that differentiates our approach from this line of work.

\section{Modeling Gendered Language}

As explained in $\S 1$, our aim is quantify the degree to which the language used to describe men and women is different and, moreover, different in a positive or negative way. To do this, we therefore introduce a generative latent-variable model that jointly represents adjective (or verb) choice, with its sentiment, given the natural gender of a head (or dependent) noun. This model, which is based on the sparse additive generative model (SAGE; Eisenstein et al., 2011), ${ }^{4}$ enables us to extract ranked lists of adjectives (or verbs) that are used, with particular sentiments, to describe male or female nouns.

We define $\mathcal{G}$ to be the set of gendered, animate nouns in our corpus and $n \in \mathcal{G}$ to be one such noun. We represent $n$ via a multi-hot vector $\boldsymbol{f}_{n} \in\{0,1\}^{T}$ of its lexical features-i.e., its genderless lemma, its gender (male or female), and its number (singular or plural). In other words, $\boldsymbol{f}_{n}$ always has exactly three non-zero entries; for example, the only non-zero entries of $f_{\text {stewardesses }}$ are those corresponding to STEWARD, +FEM, and +PL. We define $\mathcal{V}$ to be the set of adjectives (or verbs) in our corpus and $\nu \in \mathcal{V}$ to be one such adjective (or verb). To simplify exposition, we refer to each adjective (or verb) that attaches to noun $n$ as a neighbor of $n$. Finally, we define $\mathcal{S}=\{$ POS, NEG, NEU $\}$ to be a set of three sentiments and $s \in \mathcal{S}$ to be one such sentiment.

Drawing inspiration from SAGE, our model jointly represents nouns, neighbors, and (latent)

\footnotetext{
${ }^{4} \mathrm{SAGE}$ is a flexible alternative to latent Dirichlet allocation (LDA; Blei et al., 2003) — the most widely used statistical topic model. Our study could also have been conducted using LDA; drawing on SAGE was primarily a matter of personal taste.
}

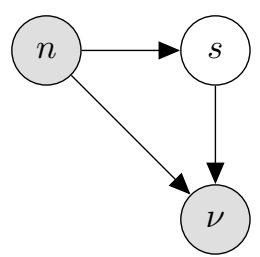

Figure 3: Graphical model depicting our model's representation of nouns, neighbors, and (latent) sentiments.

sentiments as depicted in Fig. 3. Specifically,

$$
p(\nu, n, s)=p(\nu \mid s, n) p(s \mid n) p(n) .
$$

The first factor in eq. (1) is defined as

$$
p(\nu \mid s, n) \propto \exp \left\{m_{\nu}+\boldsymbol{f}_{n}^{\top} \boldsymbol{\eta}(\nu, s)\right\},
$$

where $\boldsymbol{m} \in \mathbb{R}^{|\mathcal{V}|}$ is a background distribution and $\boldsymbol{\eta}(\nu, s) \in \mathbb{R}^{T}$ is a neighbor- and sentiment-specific deviation. The second factor in eq. (1) is defined as

$$
p(s \mid n) \propto \exp \left(\omega_{s}^{n}\right),
$$

where $\omega_{s}^{n} \in \mathbb{R}$, while the third factor is defined as

$$
p(n) \propto \exp \left(\xi_{n}\right),
$$

where $\xi_{n} \in \mathbb{R}$. We can then extract lists of neighbors that are used, with particular sentiments, to describe male and female nouns, ranked by scores that are a function of their deviations. For example, the score for neighbor $\nu$ when used, with positive sentiment, to describe a male noun is defined as

$$
\tau_{\text {MASC-POS }}(\nu) \propto \exp \left\{\boldsymbol{g}_{\text {MASC }}^{\top} \boldsymbol{\eta}(\nu, \text { POS })\right\},
$$

where $\boldsymbol{g}_{\text {MASC }} \in\{0,1\}^{T}$ is a vector where only the entry that corresponds to +MASC is non-zero.

Because our corpus does not contain explicit sentiment information, we marginalize out $s$ :

$$
p(\nu, n)=\sum_{s \in \mathcal{S}} p(\nu \mid s, n) p(s \mid n) p(n) .
$$

This yields the following objective function:

$$
\sum_{n \in \mathcal{G}} \sum_{\nu \in \mathcal{V}} \hat{p}(\nu, n) \log (p(\nu, n))
$$

where $\hat{p}(\nu, n) \propto \#(\nu, n)$ is the empirical probability of neighbor $\nu$ and noun $n$ in our corpus.

To ensure that the latent variables in our model correspond to positive, negative, and neutral sentiments, we rely on posterior regularization (Ganchev et al., 2010). Given an additional distribution $q(s \mid \nu)$ that provides external information 
about the sentiment of neighbor $\nu$, we regularize $p(s \mid \nu)$, as defined by our model, to be close (in the sense of KL-divergence) to $q(s \mid \nu)$. Specifically, we construct the following posterior regularizer:

$$
\begin{aligned}
& R_{\text {post }} \\
& \quad=\operatorname{KL}(q(s \mid \nu) \| p(s \mid \nu)) \\
& =-\sum_{s \in \mathcal{S}} q(s \mid \nu) \log (p(s \mid \nu))+H(q),
\end{aligned}
$$

where $H(q)$ is constant and $p(s \mid \nu)$ is defined as

$$
\begin{aligned}
p(s \mid \nu) & =\sum_{n \in \mathcal{G}} p(s, n \mid \nu) \\
& =\sum_{n \in \mathcal{G}} \frac{p(\nu \mid n, s) p(s \mid n) p(n)}{p(\nu)} .
\end{aligned}
$$

We use the combined sentiment lexicon of Hoyle et al. (2019) as $q(s \mid \nu)$. This lexicon represents each word's sentiment as a three-dimensional Dirichlet distribution, thereby accounting for the relative confidence in the strength of each sentiment and, in turn, accommodating polysemous and rare words. By using the lexicon as external information in our posterior regularizer, we can control the extent to which it influences the latent variables.

We add the regularizer in eq. (8) to the objective function in eq. (7), using a multiplier $\beta$ to control the strength of the posterior regularization. We also impose an $L_{1}$-regularizer $\alpha \cdot\|\boldsymbol{\eta}\|_{1}$ to induce sparsity. The complete objective function is then

$$
\begin{gathered}
\sum_{n \in \mathcal{G}} \sum_{\nu \in \mathcal{V}} \hat{p}(\nu, n) \log (p(\nu, n)) \\
+\alpha \cdot\|\boldsymbol{\eta}\|_{1}+\beta \cdot R_{\text {post }} .
\end{gathered}
$$

We optimize eq. (12) with respect to $\boldsymbol{\eta}(\cdot, \cdot), \boldsymbol{\omega}$, and $\boldsymbol{\xi}$ using the Adam optimizer (Kingma and $\mathrm{Ba}$, 2015) with $\alpha$ and $\beta$ set as described in $\S 4$. To ensure that the parameters are interpretable (e.g., to avoid a negative $\boldsymbol{\eta}$ (PREGNANT, NEG) canceling out a positive $\boldsymbol{\eta}$ (PREGNANT, POS)) , we also constrain $\boldsymbol{\eta}(\cdot, \cdot)$ to be non-negative, although without this constraint, our results are largely the same.

Relationship to pointwise mutual information. Our model also recovers pointwise mutual information (PMI), which has been used previously to identify gendered language (Rudinger et al., 2017).

Proposition 1. Consider the following restricted version of our model. Let $\boldsymbol{f}_{g} \in\{0,1\}^{2}$ be a onehot vector that represents only the gender of a noun $n$. We write $g$ instead of $n$, equivalence-classing all nouns as either MASC or FEM. Let $\boldsymbol{\eta}^{\star}(\cdot): \mathcal{V} \rightarrow \mathbb{R}^{2}$ be the maximum-likelihood estimate for the special case of our model without (latent) sentiments:

$$
p(\nu \mid g) \propto \exp \left(m_{\nu}+\boldsymbol{f}_{g}^{\top} \boldsymbol{\eta}^{\star}(\nu)\right) .
$$

Then, we have

$$
\tau_{g}(\nu) \propto \exp (P M I(\nu, g)) .
$$

Proof. See App. B.

Proposition 1 says that if we use a limited set of lexical features (i.e., only gender) and estimate our model without any regularization or latent sentiments, then ranking the neighbors by $\tau_{g}(\nu)$ (i.e., by their deviations from the background distribution) is equivalent to ranking them by their PMI. This proposition therefore provides insight into how our model builds on PMI. Specifically, in contrast to PMI, 1) our model can consider lexical features other than gender, 2) our model is regularized to avoid the pitfalls of maximumlikelihood estimation, and 3) our model cleanly incorporates latent sentiments, relying on posterior regularization to ensure that the $p(s \mid \nu)$ is close to the sentiment lexicon of Hoyle et al. (2019).

\section{Experiments, Results, and Discussion}

We use our model to study the corpus of Goldberg and Orwant (2013) by running it separately on the noun-adjectives pairs, the NSUBJ-verb pairs, and the DOBJ-verb pairs. We provide a full list of the lemmatized, gendered, animate nouns in App. A. We use $\alpha \in\left\{0,10^{-5}, 10^{-4}, 0.001,0.01\right\}$ and $\beta \in\left\{10^{-5}, 10^{-4}, 0.001,0.01,0.1,1,10,100\right\}$; when we report results below, we use parameter values averaged over these hyperparameter settings.

\subsection{Q1: Qualitative Differences}

Our first research question concerns the qualitative differences between the language used to describe men and women. To answer this question, we use our model to extract ranked lists of neighbors that are used, with particular sentiments, to describe male and female nouns. As explained in $\S 3$, we rank the neighbors by their deviations from the background distribution (see, for example, eq. (5)).

Qualitative evaluation. In Tab. 2, we provide, for each sentiment, the 25 largest-deviation adjectives used to describe male and female nouns. The 


\begin{tabular}{|c|c|c|c|c|c|c|c|c|c|c|c|}
\hline \multicolumn{2}{|c|}{$\tau_{\text {MASC-POS }}$} & \multicolumn{2}{|c|}{$\tau_{\text {MASC-NEG }}$} & \multicolumn{2}{|c|}{$\tau_{\text {MASC-NEU }}$} & \multicolumn{2}{|c|}{$\tau_{\text {FEM-POS }}$} & \multicolumn{2}{|c|}{$\tau_{\text {FEM-NEG }}$} & \multicolumn{2}{|c|}{$\tau_{\text {FEM-NEU }}$} \\
\hline Adj. & Value & Adj. & Value & Adj. & Value & Adj. & Value & Adj. & Value & Adj. & Value \\
\hline faithful & 2.3 & unjust & 2.4 & german & 1.9 & pretty & 3.3 & horrible & 1.8 & virgin & 2.8 \\
\hline responsible & 2.2 & dumb & 2.3 & teutonic & 0.8 & fair & 3.3 & destructive & 0.8 & alleged & 2.0 \\
\hline adventurous & 1.9 & violent & 1.8 & financial & 2.6 & beautiful & 3.4 & notorious & 2.6 & maiden & 2.8 \\
\hline grand & 2.6 & weak & 2.0 & feudal & 2.2 & lovely & 3.4 & dreary & 0.8 & russian & 1.9 \\
\hline worthy & 2.2 & evil & 1.9 & later & 1.6 & charming & 3.1 & ugly & 3.2 & fair & 2.6 \\
\hline brave & 2.1 & stupid & 1.6 & austrian & 1.2 & sweet & 2.7 & weird & 3.0 & widowed & 2.4 \\
\hline good & 2.3 & petty & 2.4 & feudatory & 1.8 & grand & 2.6 & harried & 2.4 & grand & 2.1 \\
\hline normal & 1.9 & brutal & 2.4 & maternal & 1.6 & stately & 3.8 & diabetic & 1.2 & byzantine & 2.6 \\
\hline ambitious & 1.6 & wicked & 2.1 & bavarian & 1.5 & attractive & 3.3 & discontented & 0.5 & fashionable & 2.5 \\
\hline gallant & 2.8 & rebellious & 2.1 & negro & 1.5 & chaste & 3.3 & infected & 2.8 & aged & 1.8 \\
\hline mighty & 2.4 & bad & 1.9 & paternal & 1.4 & virtuous & 2.7 & unmarried & 2.8 & topless & 3.9 \\
\hline loyal & 2.1 & worthless & 1.6 & frankish & 1.8 & fertile & 3.2 & unequal & 2.4 & withered & 2.9 \\
\hline valiant & 2.8 & hostile & 1.9 & welsh & 1.7 & delightful & 2.9 & widowed & 2.4 & colonial & 2.8 \\
\hline courteous & 2.6 & careless & 1.6 & ecclesiastical & 1.6 & gentle & 2.6 & unhappy & 2.4 & diabetic & 0.7 \\
\hline powerful & 2.3 & unsung & 2.4 & rural & 1.4 & privileged & 1.4 & horrid & 2.2 & burlesque & 2.9 \\
\hline rational & 2.1 & abusive & 1.5 & persian & 1.4 & romantic & 3.1 & pitiful & 0.8 & blonde & 2.9 \\
\hline supreme & 1.9 & financial & 3.6 & belted & 1.4 & enchanted & 3.0 & frightful & 0.5 & parisian & 2.7 \\
\hline meritorious & 1.5 & feudal & 2.5 & swiss & 1.3 & kindly & 3.2 & artificial & 3.2 & clad & 2.5 \\
\hline serene & 1.4 & false & 2.3 & finnish & 1.1 & elegant & 2.8 & sullen & 3.1 & female & 2.3 \\
\hline godlike & 2.3 & feeble & 1.9 & national & 2.2 & dear & 2.2 & hysterical & 2.8 & oriental & 2.2 \\
\hline noble & 2.3 & impotent & 1.7 & priestly & 1.8 & devoted & 2.0 & awful & 2.6 & ancient & 1.7 \\
\hline rightful & 1.9 & dishonest & 1.6 & merovingian & 1.6 & beauteous & 3.9 & haughty & 2.6 & feminist & 2.9 \\
\hline eager & 1.9 & ungrateful & 1.5 & capetian & 1.4 & sprightly & 3.2 & terrible & 2.4 & matronly & 2.6 \\
\hline financial & 3.3 & unfaithful & 2.6 & prussian & 1.4 & beloved & 2.5 & damned & 2.4 & pretty & 2.5 \\
\hline chivalrous & 2.6 & incompetent & 1.7 & racial & 0.9 & pleasant & 1.8 & topless & 3.5 & asiatic & 2.0 \\
\hline
\end{tabular}

Table 2: For each sentiment, we provide the largest-deviation adjectives used to describe male and female nouns.

results are striking: it is immediately apparent that positive adjectives describing women are often related to their appearance (e.g., beautiful, fair, and pretty). Sociolinguistic studies of other corpora, such as British newspapers (Caldas-Coulthard and Moon, 2010), have also revealed this pattern. Adjectives relating to fertility, such as fertile and barren, are also more prevalent for women. We provide similar tables for verbs in App. D. Negative verbs describing men are often related to violence (e.g., murder, fight, kill, and threaten). Meanwhile, women are almost always the object of rape, which aligns with our knowledge of the world and supports the collocation of rape and girl found by Baker (2014). Broadly speaking, positive verbs describing men tend to connote virtuosity (e.g., gallant and inspire), while those describing women appear more trivial (e.g., sprightly, giggle, and kiss).

Correlation with human judgments. To determine whether the output from our model accords with previous human judgements of gender stereotypes, we use the corpus of Williams and Bennett (1975), which consists of 63 adjectives annotated with (binary) gender stereotypes. We measure Spearman's $\rho$ between these annotations and the probabilities output by our model. We find a relatively strong positive correlation of $\rho=0.59$ $\left(p<10^{-6}\right.$ ), which indicates that the output from our model aligns with common gender stereotypes captured by previous human judgements. We also measure the correlation between continuous annotations of 300 adjectives from two follow-up studies (Williams and Best, 1990, 1977) ${ }^{5}$ and the probabilities output by our model. Here, the correlation is $\rho=0.33\left(p<10^{-8}\right)$, and the binarized annotations agree with the output from our model for $64 \%$ of terms. We note that some of the disagreement is due to reporting bias (Gordon and Van Durme, 2013) in our corpus. For example, only men are described in our corpus as effeminate, although humans judge it to be a highly feminine adjective.

\subsection{Q2: Quantitative differences}

Our second research question concerns the quantitative differences between the language used to describe men and women. To answer this question, we use two existing semantic resources-one for adjectives (Tsvetkov et al., 2014) and one for verbs (Miller et al., 1993) - to quantify the patterns revealed by our model. Again, we use our model to extract ranked lists of neighbors that are used, with particular sentiments, to describe male and female nouns. We consider only the 200 largest-deviation

\footnotetext{
${ }^{5}$ The studies consider the same set of words 20 years apart; we average their annotations, obtained from Garg et al. (2018).
} 


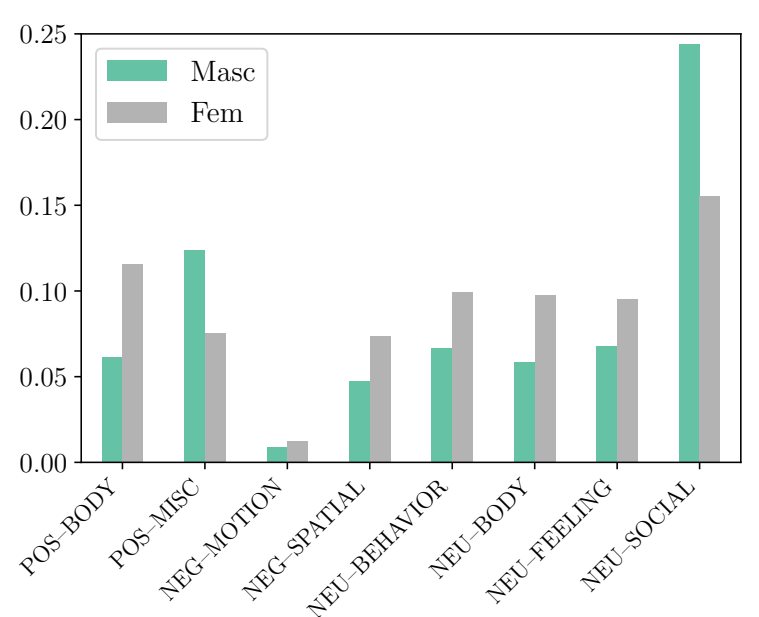

Figure 4: The frequency with which the 200 largestdeviation adjectives for each sentiment and gender correspond to each sense from Tsvetkov et al. (2014).

neighbors for each sentiment and gender. This restriction allows us to perform an unpaired permutation test (Good, 2004) to determine whether there are significant differences between the language used to describe men and women.

Adjective evaluation. Women are supposedly more often described using adjectives related to their bodies and emotions. For example, de Beauvoir (1953) writes that "from girlhood, women are socialized to live and experience their bodies as objects for another's gaze..." Although studies of reasonably large corpora have found evidence to support this supposition (Norberg, 2016), none have done so at scale with statistical significance testing. We use the semantic resource of Tsvetkov et al. (2014), which categorizes adjectives into thirteen senses: BEHAVIOR, BODY, FEELING, MIND, etc. Specifically, each adjective has a distribution over senses, capturing how often the adjective corresponds to each sense. We analyze the largestdeviation adjectives for each sentiment and gender by computing the frequency with which these adjectives correspond to each sense. We depict these frequencies in Fig. 4. Specifically, we provide frequencies for the senses where, after Bonferroni correction, the differences between men and women are significant. We find that adjectives used to describe women are indeed more often related to their bodies and emotions than adjectives used to describe men.

Verb evaluation. To evalaute verbs senses, we take the same approach as for adjectives. We use the semantic resource of Miller et al. (1993), which

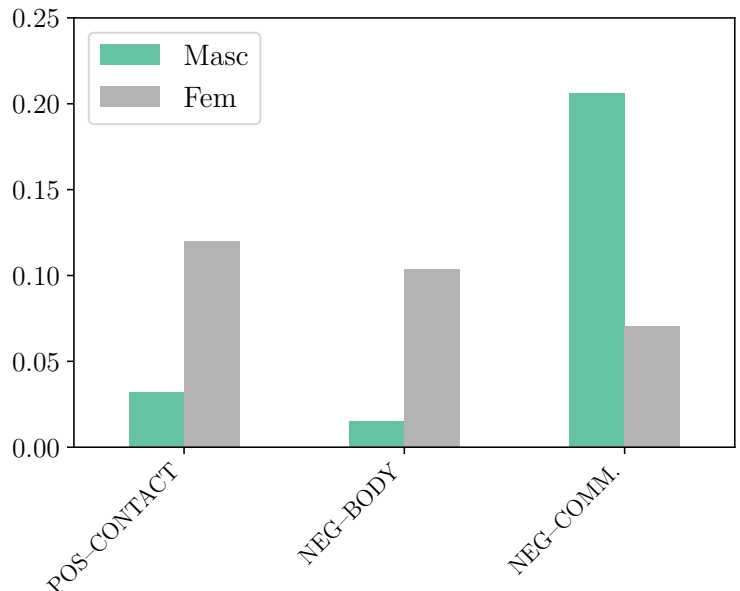

Figure 5: The frequency with which the 200 largestdeviation verbs for each sentiment and gender correspond to each sense from Miller et al. (1993). These results are only for the NSUBJ-verb pairs; there are no statistically significant differences for DOBJ-verb pairs.

\begin{tabular}{lllllllll}
\hline & \multicolumn{2}{c}{ ADJ } & & \multicolumn{2}{c}{ NSUBJ } & & \multicolumn{2}{c}{ DOBJ } \\
\cline { 2 - 3 } & MSC & FEM & & MSC & FEM & & MSC & FEM \\
\hline POS & 0.34 & 0.38 & & 0.37 & 0.36 & & 0.37 & 0.36 \\
NEG & 0.30 & 0.31 & & 0.33 & 0.34 & & 0.34 & 0.35 \\
NEU & $\mathbf{0 . 3 6}$ & $\mathbf{0 . 3 1}$ & & 0.30 & 0.30 & & 0.30 & 0.29 \\
\hline
\end{tabular}

Table 3: The frequency with which the 200 largestdeviation neighbors for each gender correspond to each sentiment, obtained using a simplified version of our model and the lexicon of Hoyle et al. (2019). Significant differences ( $p<0.05 / 3$ under an unpaired permutation test with Bonferroni correction) are in bold.

categorizes verbs into fifteen senses. Each verb has a distribution over senses, capturing how often the verb corresponds to each sense. We consider two cases: the NSUBJ-verb pairs and the DOBJ-verb pairs. Overall, there are fewer significant differences for verbs than there are for adjectives. There are no statistically significant differences for the DOBJ-verb pairs. We depict the results for the NSUBJ-verb pairs in Fig. 5. We find that verbs used to describe women are more often related to their bodies than verbs used to describe men.

\subsection{Q3: Differences in sentiment}

Our final research question concerns the overall sentiment of the language used to describe men and women. To answer this question, we use a simplified version of our model, without the latent sentiment variables or the posterior regularizer. We are then able to use the combined sentiment lexicon of Hoyle et al. (2019) to analyze the largest-deviation 
neighbors for each gender by computing the frequency with which each neighbor corresponds to each sentiment. We report these frequencies in Tab. 3. We find that there is only one significant difference: adjectives used to describe men are more often neutral than those used to describe women.

\section{Conclusion and Limitations}

We presented an experimental framework for quantitatively studying the ways in which the language used to describe men and women is different and, moreover, different in a positive or negative way. We introduced a generative latent-variable model that jointly represents adjective (or verb) choice, with its sentiment, given the natural gender of a head (or dependent) noun. Via our experiments, we found evidence in support of common gender stereotypes. For example, positive adjectives used to describe women are more often related to their bodies than adjectives used to describe men. Our study has a few limitations that we wish to highlight. First, we ignore demographics (e.g., age, gender, location) of the speaker, even though such demographics are likely influence word choice. Second, we ignore genre (e.g., news, romance) of the text, even though genre is also likely to influence the language used to describe men and women. In addition, depictions of men and women have certainly changed over the period covered by our corpus; indeed, Underwood et al. (2018) found evidence of such a change for fictional characters. In future work, we intend to conduct a diachronic analysis in English using the same corpus, in addition to a cross-linguistic study of gendered language.

\section{Acknowledgments}

We would like to thank the three anonymous ACL 2019 reviewers for their comments on the submitted version, as well as the anonymous reviewers of a previous submission. We would also like to thank Adina Williams and Eleanor Chodroff for their comments on versions of the manuscript. The last author would like to acknowledge a Facebook fellowship.

\section{References}

Paul Baker. 2005. Public discourses of gay men. Routledge.

Paul Baker. 2013. Introduction: Virtual special issue of gender and language on corpus approaches. Gender and Language, 1(1).

Paul Baker. 2014. Using corpora to analyze gender. A\&C Black.

David Bamman, Jacob Eisenstein, and Tyler Schnoebelen. 2014. Gender identity and lexical variation in social media. Journal of Sociolinguistics, 18(2):135-160.

Simone de Beauvoir. 1953. The second sex.

David M. Blei, Andrew Y. Ng, and Michael I. Jordan. 2003. Latent Dirichlet allocation. Journal of $\mathrm{Ma}$ chine Learning Research, 3(Jan):993-1022.

Tolga Bolukbasi, Kai-Wei Chang, James Y. Zou, Venkatesh Saligrama, and Adam T. Kalai. 2016. Man is to computer programmer as woman is to homemaker? Debiasing word embeddings. In $\mathrm{Ad}$ vances in Neural Information Processing Systems, pages 4349-4357.

Carmen Rosa Caldas-Coulthard and Rosamund Moon. 2010. 'Curvy, hunky, kinky': Using corpora as tools for critical analysis. Discourse \& Society, 21(2):99133.

Jacob Eisenstein, Amr Ahmed, and Eric P Xing. 2011. Sparse Additive Generative Models of Text. page 8.

Kuzman Ganchev, Jennifer Gillenwater, Ben Taskar, et al. 2010. Posterior regularization for structured latent variable models. Journal of Machine Learning Research, 11(Jul):2001-2049.

Nikhil Garg, Londa Schiebinger, Dan Jurafsky, and James Zou. 2018. Word embeddings quantify 100 years of gender and ethnic stereotypes. Proceedings of the National Academy of Sciences, 115(16):E3635-E3644.

Yoav Goldberg and Jon Orwant. 2013. A dataset of syntactic-ngrams over time from a very large corpus of English books. In Second Joint Conference on Lexical and Computational Semantics (*SEM), Volume 1: Proceedings of the Main Conference and the Shared Task: Semantic Textual Similarity, pages 241-247, Atlanta, Georgia, USA. Association for Computational Linguistics.

Phillip I. Good. 2004. Permutation, parametric, and bootstrap tests of hypotheses.

Jonathan Gordon and Benjamin Van Durme. 2013. Reporting Bias and Knowledge Acquisition. In Proceedings of the 2013 Workshop on Automated Knowledge Base Construction, AKBC '13, pages 25-30, New York, NY, USA. ACM.

Alexander Hoyle, Lawrence Wolf-Sonkin, Hanna Wallach, Ryan Cotterell, and Isabelle Augenstein. 2019. Combining disparate sentiment lexica with a multiview variational autoencoder. In Proceedings of the 2019 Conference of the North American Chapter of 
the Association for Computational Linguistics: $\mathrm{Hu}$ man Language Technologies, Volume 2 (Short Papers). Association for Computational Linguistics.

Diederik P. Kingma and Jimmy Ba. 2015. Adam: A method for stochastic optimization. In International Conference on Learning Representations (ICLR).

Robin Lakoff. 1973. Language and woman's place. Language in Society, 2(1):45-79.

John P. McKee and Alex C. Sherriffs. 1957. The differential evaluation of males and females. Journal of Personality, 25(3):356-371.

George A. Miller, Claudia Leacock, Randee Tengi, and Ross T. Bunker. 1993. A semantic concordance. In Proceedings of the workshop on Human Language Technology (HLT), pages 303-308. Association for Computational Linguistics.

Moon, Rosamund. 2014. From gorgeous to grumpy: Adjectives, age, and gender. Gender and Language, $8(1): 5-41$.

Cathrine Norberg. 2016. Naughty Boys and Sexy Girls: The Representation of Young Individuals in a WebBased Corpus of English. Journal of English Linguistics, 44(4):291-317.

Cathy O'Neil. 2016. Weapons of math destruction: How big data increases inequality and threatens democracy. Broadway Books.

Margaret Ott. 2016. Tweet like a girl: Corpus analysis of gendered language in social media.

Michael Pearce. 2008. Investigating the collocational behaviour of man and woman in the BNC using Sketch Engine. Corpora, 3(1):1-29.

Jeffrey Z. Rubin, Frank J. Provenzano, and Zella Luria. 1974. The eye of the beholder: Parents' views on sex of newborns. American Journal of Orthopsychiatry, 44(4):512.

Rachel Rudinger, Chandler May, and Benjamin Van Durme. 2017. Social bias in elicited natural language inferences. In Proceedings of the First ACL Workshop on Ethics in Natural Language Processing, pages 74-79.

Rachel Rudinger, Aaron Steven White, and Benjamin Van Durme. 2018. Neural models of factuality. In Proceedings of the 2018 Conference of the North American Chapter of the Association for Computational Linguistics: Human Language Technologies, Volume 1 (Long Papers), pages 731-744. Association for Computational Linguistics.

Alexandra Schofield and Leo Mehr. 2016. Genderdistinguishing features in film dialogue. In Proceedings of the Fifth Workshop on Computational Linguistics for Literature, pages 32-39, San Diego, California, USA. Association for Computational Linguistics.
Daniel Storage, Zachary Horne, Andrei Cimpian, and Sarah-Jane Leslie. 2016. The frequency of "brilliant" and "genius" in teaching evaluations predicts the representation of women and African Americans across fields. PloS one, 11(3):e0150194.

Yulia Tsvetkov, Nathan Schneider, Dirk Hovy, Archna Bhatia, Manaal Faruqui, and Chris Dyer. 2014. Augmenting English adjective senses with supersenses. In Proceedings of the Ninth International Conference on Language Resources and Evaluation (LREC'14), Reykjavik, Iceland. European Language Resources Association (ELRA).

Ted Underwood, David Bamman, and Sabrina Lee. 2018. The transformation of gender in englishlanguage fiction.

John E. Williams and Susan M. Bennett. 1975. The definition of sex stereotypes via the adjective check list. Sex Roles, 1(4):327-337.

John E. Williams and Deborah L. Best. 1977. Sex Stereotypes and Trait Favorability on the Adjective Check List. Educational and Psychological Measurement, 37(1):101-110.

John E. Williams and Deborah L. Best. 1990. Measuring sex stereotypes: a multination study. Newbury Park, Calif. : Sage.

Jieyu Zhao, Tianlu Wang, Mark Yatskar, Vicente Ordonez, and Kai-Wei Chang. 2017. Men also like shopping: Reducing gender bias amplification using corpus-level constraints. In Proceedings of the 2017 Conference on Empirical Methods in Natural Language Processing, pages 2979-2989, Copenhagen, Denmark. Association for Computational Linguistics.

Jieyu Zhao, Tianlu Wang, Mark Yatskar, Vicente Ordonez, and Kai-Wei Chang. 2018. Gender bias in coreference resolution: Evaluation and debiasing methods. In Proceedings of the 2018 Conference of the North American Chapter of the Association for Computational Linguistics: Human Language Technologies, Volume 2 (Short Papers), pages 15-20. Association for Computational Linguistics. 


\section{A List of Gendered, Animate Nouns}

Tab. 4 contains the full list of gendered, animate nouns that we use. We consider each row in this table to be the inflected forms of a single lemma.

\begin{tabular}{llll}
\hline \multicolumn{2}{c}{ Male } & \multicolumn{2}{c}{ Female } \\
Singular & Plural & Singular & Plural \\
\hline man & men & woman & women \\
boy & boys & girl & girls \\
father & fathers & mother & mothers \\
son & sons & daughter & daughters \\
brother & brothers & sister & sisters \\
husband & husbands & wife & wives \\
uncle & uncles & aunt & aunts \\
nephew & nephews & niece & nieces \\
emperor & emperors & empress & empresses \\
king & kings & queen & queens \\
prince & princes & princess & princesses \\
duke & dukes & duchess & duchesses \\
lord & lords & lady & ladies \\
knight & knights & dame & dames \\
waiter & waiters & waitress & waitresses \\
actor & actors & actress & actresses \\
god & gods & goddess & goddesses \\
policeman & policemen & policewoman & policewomen \\
postman & postmen & postwoman & postwomen \\
hero & heros & heroine & heroines \\
wizard & wizards & witch & witches \\
steward & stewards & stewardess & stewardesses \\
he & - & she & - \\
\hline
\end{tabular}

Table 4: Gendered, animate nouns.

\section{B Relationship to PMI}

Proposition 1. Consider the following restricted version of our model. Let $\boldsymbol{f}_{g} \in\{0,1\}^{2}$ be a onehot vector that represents only the gender of a noun. We write $g$ instead of $n$, equivalence-classing all nouns as either MASC or FEM. Let $\boldsymbol{\eta}^{\star}(\cdot): \mathcal{V} \rightarrow \mathbb{R}^{2}$ be the maximum-likelihood estimate for the special case of our model without (latent) sentiments:

$$
p(\nu \mid g) \propto \exp \left(m_{\nu}+\boldsymbol{f}_{g}^{\top} \boldsymbol{\eta}^{\star}(\nu)\right) .
$$

Then, we have

$$
\tau_{g}(\nu) \propto \exp (P M I(\nu, g)) .
$$

Proof. First, we note our model has enough parameters to fit the empirical distribution exactly:

$$
\begin{aligned}
\hat{p}(\nu \mid g) & =p(\nu \mid g) \\
& \propto \exp \left\{m_{\nu}+\boldsymbol{f}_{g}^{\top} \boldsymbol{\eta}^{\star}(\nu)\right\} .
\end{aligned}
$$

Then, we proceed with an algebraic manipulation of the definition of pointwise mutual information:

$$
\begin{aligned}
\operatorname{PMI}(\nu, g) & =\log \frac{\hat{p}(\nu, n)}{\hat{p}(\nu) \hat{p}(n)} \\
& =\log \frac{\hat{p}(\nu \mid n)}{\hat{p}(\nu)} \\
& =\log \frac{p(\nu \mid n)}{\hat{p}(\nu)} \\
& =\log \frac{p(\nu \mid n)}{\exp \left\{m_{\nu}\right\}} \\
& =\log \frac{1}{Z} \frac{\exp \left\{m_{\nu}+\boldsymbol{f}_{g}^{\top} \boldsymbol{\eta}^{\star}(\nu)\right\}}{\exp \left\{m_{\nu}\right\}} \\
& =\log \frac{1}{Z} \exp \left\{\boldsymbol{f}_{g}^{\top} \boldsymbol{\eta}^{\star}(\nu)\right\} \\
& =\boldsymbol{f}_{g}^{\top} \boldsymbol{\eta}^{\star}(\nu)-\log Z .
\end{aligned}
$$

Now we have

$$
\begin{aligned}
\tau_{g}(\nu) & \propto \exp \left\{f_{g}^{\top} \boldsymbol{\eta}^{\star}(\nu)\right\} \\
& \propto \exp \left\{f_{g}^{\top} \boldsymbol{\eta}^{\star}(\nu)-\log Z\right\} \\
& =\exp (P M I(\nu, g)),
\end{aligned}
$$

which is what we wanted to show.

\section{Senses}

In Tab. 5, we list the senses for adjectives (Tsvetkov et al., 2014) and for verbs (Miller et al., 1993).

\begin{tabular}{ll}
\hline Adjectives & Verbs \\
\hline Behavior & Body \\
Body & Change \\
Feeling & Cognition \\
Mind & Communication \\
Miscellaneous & Competition \\
Motion & Consumption \\
Perception & Contact \\
Quantity & Creation \\
Social & Emotion \\
Spatial & Motion \\
Substance & Perception \\
Temporal & Possession \\
Weather & Social \\
& Stative \\
& Weather \\
\hline
\end{tabular}

Table 5: Senses for adjectives and verbs.

\section{Additional Results}

In Tab. 6 and Tab. 7, we provide the largestdeviation verbs used to describe male and female nouns for NSUBJ-verb pairs and DOBJ-verb pairs. 


\begin{tabular}{|c|c|c|c|c|c|c|c|c|c|c|c|}
\hline \multicolumn{2}{|c|}{$\tau_{\text {MASC-POS }}$} & \multicolumn{2}{|c|}{$\tau_{\text {MASC-NEG }}$} & \multicolumn{2}{|c|}{$\tau_{\text {MASC-NEU }}$} & \multicolumn{2}{|l|}{$\tau_{\text {FEM-POS }}$} & \multicolumn{2}{|c|}{$\tau_{\text {FEM-NEG }}$} & \multicolumn{2}{|c|}{$\tau_{\text {FEM-NEU }}$} \\
\hline Verb & Value & Verb & Value & Verb & Value & Verb & Value & Verb & Value & Verb & Value \\
\hline succeed & 1.6 & fight & 1.2 & extend & 0.7 & celebrate & 2.4 & persecute & 2.1 & faint & 0.7 \\
\hline protect & 1.4 & fail & 1.0 & found & 0.8 & fascinate & 0.8 & faint & 1.0 & be & 1.1 \\
\hline favor & 1.3 & fear & 1.0 & strike & 1.3 & facilitate & 0.7 & fly & 1.0 & go & 0.4 \\
\hline flourish & 1.3 & murder & 1.5 & own & 1.1 & marry & 1.8 & weep & 2.3 & find & 0.1 \\
\hline prosper & 1.7 & shock & 1.6 & collect & 1.1 & smile & 1.8 & harm & 2.2 & fly & 0.4 \\
\hline support & 1.5 & blind & 1.6 & set & 0.8 & fan & 0.8 & wear & 2.0 & fall & 0.1 \\
\hline promise & 1.5 & forbid & 1.5 & wag & 1.0 & kiss & 1.8 & mourn & 1.7 & wear & 0.9 \\
\hline welcome & 1.5 & kill & 1.3 & present & 0.9 & champion & 2.2 & gasp & 1.1 & leave & 0.7 \\
\hline favour & 1.2 & protest & 1.3 & pretend & 1.1 & adore & 2.0 & fatigue & 0.7 & fell & 0.1 \\
\hline clear & 1.9 & cheat & 1.3 & prostrate & 1.1 & dance & 1.7 & scold & 1.8 & vanish & 1.3 \\
\hline reward & 1.8 & fake & 0.8 & want & 0.9 & laugh & 1.6 & scream & 2.1 & come & 0.7 \\
\hline appeal & 1.6 & deprive & 1.5 & create & 0.9 & have & 1.4 & confess & 1.7 & fertilize & 0.6 \\
\hline encourage & 1.5 & threaten & 1.3 & pay & 1.1 & play & 1.0 & get & 0.5 & flush & 0.5 \\
\hline allow & 1.5 & frustrate & 0.9 & prompt & 1.0 & give & 0.8 & gossip & 2.0 & spin & 1.6 \\
\hline respect & 1.5 & fright & 0.9 & brazen & 1.0 & like & 1.8 & worry & 1.8 & dress & 1.4 \\
\hline comfort & 1.4 & temper & 1.4 & tarry & 0.7 & giggle & 1.4 & be & 1.3 & fill & 0.2 \\
\hline treat & 1.3 & horrify & 1.4 & front & 0.5 & extol & 0.6 & fail & 0.4 & fee & 0.2 \\
\hline brave & 1.7 & neglect & 1.4 & flush & 0.3 & compassionate & 1.9 & fight & 0.4 & extend & 0.1 \\
\hline rescue & 1.5 & argue & 1.3 & reach & 0.9 & live & 1.4 & fake & 0.3 & sniff & 1.6 \\
\hline win & 1.5 & denounce & 1.3 & escape & 0.8 & free & 0.9 & overrun & 2.4 & celebrate & 1.1 \\
\hline warm & 1.5 & concern & 1.2 & gi & 0.7 & felicitate & 0.6 & hurt & 1.8 & clap & 1.1 \\
\hline praise & 1.4 & expel & 1.7 & rush & 0.6 & mature & 2.2 & complain & 1.7 & appear & 0.9 \\
\hline fit & 1.4 & dispute & 1.5 & duplicate & 0.5 & exalt & 1.7 & lament & 1.5 & gi & 0.8 \\
\hline wish & 1.4 & obscure & 1.4 & incarnate & 0.5 & surpass & 1.7 & fertilize & 0.5 & have & 0.5 \\
\hline grant & 1.3 & damn & 1.4 & freeze & 0.5 & meet & 1.1 & feign & 0.5 & front & 0.5 \\
\hline
\end{tabular}

Table 6: The largest-deviation verbs used to describe male and female nouns for NSUBJ-verb pairs.

\begin{tabular}{|c|c|c|c|c|c|c|c|c|c|c|c|}
\hline \multicolumn{2}{|c|}{$\tau_{\text {MASC-POS }}$} & \multicolumn{2}{|c|}{$\tau_{\text {MASC-NEG }}$} & \multicolumn{2}{|l|}{$\tau_{\text {MASC-NEU }}$} & \multicolumn{2}{|c|}{$\tau_{\text {FEM-POS }}$} & \multicolumn{2}{|c|}{$\tau_{\text {FEM-NEG }}$} & \multicolumn{2}{|c|}{$\tau_{\text {FEM-NEU }}$} \\
\hline Verb & Value & Verb & Value & Verb & Value & Verb & Value & Verb & Value & Verb & Value \\
\hline praise & 1.7 & fight & 1.8 & set & 1.5 & marry & 2.3 & forbid & 1.3 & have & 1.0 \\
\hline thank & 1.7 & expel & 1.8 & pay & 1.2 & assure & 3.4 & shame & 2.5 & expose & 0.8 \\
\hline succeed & 1.7 & fear & 1.6 & escape & 0.4 & escort & 1.2 & escort & 1.3 & escort & 1.4 \\
\hline exalt & 1.2 & defeat & 2.4 & use & 2.1 & exclaim & 1.0 & exploit & 0.9 & pour & 2.1 \\
\hline reward & 1.8 & fail & 1.3 & expel & 0.9 & play & 2.7 & drag & 2.1 & marry & 1.3 \\
\hline commend & 1.7 & bribe & 1.8 & summon & 1.7 & pour & 2.6 & suffer & 2.2 & take & 1.1 \\
\hline fit & 1.4 & kill & 1.6 & speak & 1.3 & create & 2.0 & shock & 2.1 & assure & 1.6 \\
\hline glorify & 2.0 & deny & 1.5 & shop & 2.6 & have & 1.8 & fright & 2.4 & fertilize & 1.6 \\
\hline honor & 1.6 & murder & 1.7 & excommunicate & 1.3 & fertilize & 1.8 & steal & 2.0 & ask & 1.0 \\
\hline welcome & 1.9 & depose & 2.3 & direct & 1.1 & eye & 0.9 & insult & 1.8 & exclaim & 0.6 \\
\hline gentle & 1.8 & summon & 2.0 & await & 0.9 & woo & 3.3 & fertilize & 1.6 & strut & 2.3 \\
\hline inspire & 1.7 & order & 1.9 & equal & 0.4 & strut & 3.1 & violate & 2.4 & burn & 1.7 \\
\hline enrich & 1.7 & denounce & 1.7 & appoint & 1.7 & kiss & 2.6 & tease & 2.3 & rear & 1.5 \\
\hline uphold & 1.5 & deprive & 1.6 & animate & 1.1 & protect & 2.1 & terrify & 2.1 & feature & 0.9 \\
\hline appease & 1.5 & mock & 1.6 & follow & 0.7 & win & 2.0 & persecute & 2.1 & visit & 1.3 \\
\hline join & 1.4 & destroy & 1.5 & depose & 1.8 & excel & 1.6 & cry & 1.8 & saw & 1.3 \\
\hline congratulate & 1.3 & deceive & 1.7 & want & 1.1 & treat & 2.3 & expose & 1.3 & exchange & 0.8 \\
\hline extol & 1.1 & bore & 1.6 & reach & 0.9 & like & 2.2 & burn & 2.6 & shame & 1.6 \\
\hline respect & 1.7 & bully & 1.5 & found & 0.8 & entertain & 2.0 & scare & 2.0 & fade & 1.2 \\
\hline brave & 1.7 & enrage & 1.4 & exempt & 0.4 & espouse & 1.4 & frighten & 1.8 & signal & 1.2 \\
\hline greet & 1.6 & shop & 2.7 & tip & 1.8 & feature & 1.2 & distract & 2.3 & see & 1.2 \\
\hline restore & 1.5 & elect & 2.2 & elect & 1.7 & meet & 2.2 & weep & 2.3 & present & 1.0 \\
\hline clear & 1.5 & compel & 2.1 & unmake & 1.5 & wish & 1.9 & scream & 2.3 & leave & 0.8 \\
\hline excite & 1.2 & offend & 1.5 & fight & 1.2 & fondle & 1.9 & drown & 2.1 & espouse & 1.3 \\
\hline flatter & 0.9 & scold & 1.4 & prevent & 1.1 & saw & 1.8 & rape & 2.0 & want & 1.1 \\
\hline
\end{tabular}

Table 7: The largest-deviation verbs used to describe male and female nouns for DOBJ-verb pairs. 